\title{
Hybrid Petri Nets-based Flow Modeling and Application on Hybrid System
}

\author{
Abdelkader Sayah ${ }^{1}$, Mohammed Chenafa ${ }^{2}$ \\ ${ }^{1,2}$ ENP d'Oran, Laboratory of Automatics and Systems Analysis (L.A.A.S) \\ Department of Electrical Engineering, Oran, Algeria
}

\begin{abstract}
Article Info
Article history:

Received Oct 16, 2019

Revised Jan 21, 2021

Accepted Feb 11, 2021

\section{Keyword:}

Modeling Simulation.

Hybrid Petri nets

Flow management

Automatied systems

Dynamic systems
\end{abstract}

\begin{abstract}
Flow management is necessary in several application areas, in the optimization of industrial production lines, in IT to manage data flows and in the automation of industrial systems. Physical systems in general consist of continuous processes interacting with discrete processes forming a hybrid dynamic system constituted by continuous dynamic type models and discrete events. The application of the hybrid Petri nets tool in the modeling, study and performance evaluation of these systems helps to analyze the dynamic properties by acting on the parameters and the structure of the models in order to evaluate their behavior. This work is focused on the application of this tool to model a material flow management system between a rotary kiln and a clinker cooler in a production line (cement process). The implementation of the modeling and the analysis of the results obtained by simulation on a software platform (Visual Object Net ++), aims to study industrial processes with mathematical tools and to follow their behavior on software, this allows us an optimal analysis of complex systems in dangerous environments, and to try practical and effective solutions by simple means before moving on to the implementation and programming of actions that require more expensive means.
\end{abstract}

Copyright $(9) 2021$ Institute of Advanced Engineering and Science. All rights reserved.

\section{Corresponding Author:}

Sayah Abdelkader,

Department of Electrical Engineering

B.P 1523, El Mnaouer, ENPO, Oran, Algeria.

Email: abdelkader_saida@yahoo.fr

\section{INTRODUCTION}

The optimization of industrial production systems requires the introduction of automation to increase production by minimizing human intervention, especially in processes in hazardous environments that have difficult or repetitive tasks, and to improve efficiency and accuracy quality products with a beneficial cost. The automation performed by a programmed logic gives the possibility of parameterization and adjustment, to ensure the safety of the installations and the stability of these systems. These physical systems have an interaction between continuous dynamic and discrete event type models are designated hybrid dynamic systems [1-3].

Flow management in production systems requires performance study and exact modeling by powerful tools, to provide solutions to problems that have arisen during model design and performance evaluation. the application of Petri nets as a graphical modeling tool facilitates the design of complex automated systems, where extensions have been proposed to extend the scope to continuous and hybrid systems, the networks exploited in this type of management are natures continuous and time-delayed, they offer formal techniques for studying fundamental discrete events and continuous temporal behavior, generally used for the modeling of production systems [4-5, 8-9].

The Petri Nets exploited in this type of application are usually Timed and Continuous Petri Nets [10], Petri Nets and Max + Algebra [11], Hybrid Petri Nets [12], Colored Petri Nets [13]. Petri nets have been used for a very long time for the representation and study of production systems [14]. The simulation tools are built on the basis of the flow models. Lighthill and Witham [15] presented in 1955 the first flow model based on the Similarity between the flow of road traffic and the flow of fluids in ducts. 
Multiple research work in modeling and design of industrial processes has been carried out in research laboratories, using analytical methods, and verified on hybrid dynamic simulators [1, 5], where object-oriented approaches of industrial processes have developed by application of generalized and hybrid Petri nets [1,3-4].

The objective is to find a reliable model and to determine its physical properties which influence the behavior of the studied system; in general, the modeling and the simulation facilitate the study for complex industrial systems and even in harsh environments, with the realization of didactic support for researchers and students in the industrial field.

In this manuscript, we present the modeling of the flow management for a hybrid dynamic system, it is based on the control of material flow at high temperature (clincker) delivered by a feeder of raw material, feeding a rotary kiln. the semi-finished product (clinker) leaving the rotary kiln at high temperature reaches $900{ }^{\circ} \mathrm{C}$, then cooled rapidly by a flow of ambient air in a cooler which also ensures continuous evacuation according to the feed rate of the rotary kiln, to storage at a temperature reduced to $100{ }^{\circ} \mathrm{C}$ [16].

The evacuation system is carried out with a variable speed according to the pressure value measured in the enclosure of the cooler, in order to avoid the overflow of filled material and to ensure the maintenance of a minimal pile which protects the high temperature equipment of the clincker, the example proposed is verified by simulation with the Visual Object Net ++ software running under Windows system, this simulation allowed us to analyze and evaluate the performance of these models by acting on the structure and adjustment parameters to ensure optimal regulation, by exploiting the potential of hybrid Petri nets in the modeling of dynamic systems. Then the results are validated by the choice of the structure of the final model.

\section{Modeling of dynamic systems by hybrid petri nets.}

The application of Petri nets allows to model and analyze the principles of behavior of systems such as synchronization, parallelism, and the sharing of resources [1, 5], as it allows the qualitative and quantitative analysis when we talk about timed networks [4].The modeling of dynamic systems with discrete and continuous systems proves their needs taking into account the rapid growth of accessible states that determines the properties of discrete physical systems, and limit modeling tools [5,8]. As a result, this extension has made it possible to extend their use to continuous and complex dynamical systems, so-called hybrid petri nets $[1,2,4]$.

Hybrid Petri nets are represented by a set of places and transitions for continuous and discrete systems, they are symbolized with doubled places for the continuous system and a simple line for the discrete system.In continuous transitions, flow velocity is represented by equations, so in discrete transitions the controls can be set with the possibility of setting the time before starting this flow. The behavior of continuous networks is realized by continuous transitions. It is different from commutations in discrete transitions.

In continuous Petri nets the marking is defined by a positive real number, the crossing of the continuous transitions is represented by a speed $V i$ which defines the number of marks crossed per unit of time, and in the discrete transitions by delays (di) [6]. This crossing is managed by a law of the dynamics of the studied system. We can associate the duration at each place of a Petri net for (P-timed), and also at each transition for (T-timed) [6].

\subsection{Continuous Hybrid Petri nets timed 2.1.1. Rules and definitions:}

Definition 1: A marked hybrid Petri net is a 7 tuple $\left(H=P, T\right.$, Pré, Post, $h$, Tempo, $\left.M_{0}\right)$, that meets the following conditions:

- $\quad P=\left\{P_{1}, P_{2}, \ldots P_{n}\right\}$ is a finite, non-empty set of places

- $\quad T=\left\{T_{1}, T_{2}, \ldots T_{m}\right\}$ is a finite set, not empty of transitions

- $\quad P \cap T=\varnothing$, that is, $\mathrm{P}$ and $\mathrm{T}$ are disjoint

- Pre: $P \times T \rightarrow\{0,1\}$ is the input incidence location

- Post: $P \times T \rightarrow\{0,1\}$ is the output incidence location

- $\quad h: P \cup T \rightarrow\{C, D\}$, called hybrid function, it indicates for each node if it is: a discrete node (D) or a continuous node (C).

- $M_{0}$, is the initial marking

Pre and Postmappings must meet the following criteria:

If $P_{i}$ et $T_{j}$ are such that $h\left(P_{i}\right)=\operatorname{Dand} h\left(T_{j}\right)=C$, then $\operatorname{Pr} e\left(P_{i}, T_{j}\right)=\operatorname{Post}\left(P_{i}, T_{j}\right)$ must be checked. 
An invariant state behavior in a hybrid petri net describes a period of time where:

- The marking of discrete places is constant.

- The vector of shooting speeds in discrete transitions, are constant.

- The vector of the reserved tokens in the discrete and continuous places is constant.

This Hybrid Petri Net behavior changes state if any of the following events occur:

- A shot of a discrete transition.

- The unreserved marking of a continuous place becomes equal to zero

- A discrete transition becomes active [4].

$V: T \rightarrow R^{+} \cup\{\infty\}$, is a function that associates a maximum fire rate in each transition.

The initial marking $M_{0}$ is a positive or zero real number vector, it can be written: $M_{0}=M(0)$, and $M(t)$ denotes the marking at time $\mathrm{t}$.

The incidence matrix $W$ is equal to: $W=($ Post - Pre $)$, it is used to determine the evolution of the Petri net. This evolution is given by the following equation [8]:

$$
d_{m} / d_{t}=W \times v(t)
$$

$v(t):$ is the vector of instantaneous fire speeds.

Either a continuous Petri R net with constant speeds:

Definition 2 a place $P_{i}$ of $R$ is fed if there is at least one transition $T_{j}$ among the input transitions of $P_{i}$ that is validated. Formally, $P_{i}$ is fed if :

$$
\sum_{T_{j} \in{ }^{\circ} P_{i}} \operatorname{Post}\left(P_{i}, T_{j}\right) v_{i}>0
$$

Definition 3 a transition is valid if each input place of $\mathrm{Tj}$ satisfies at least one of the following two conditions:

$$
\begin{aligned}
& \text { 1. } m_{i}(t)>0 \\
& \text { 2. } P_{i} \text { is fed }
\end{aligned}
$$

\subsection{Crossing speed of a transition}

Note:

$I_{i}=\operatorname{Pré}\left(P_{i}\right)=\sum_{T_{k} \in^{\circ} P_{i}} \operatorname{Post}\left(P_{i}, T_{k}\right) \cdot v_{k}$, feeding speed of the place $P_{i}$

$O_{i}=\operatorname{Post}\left(P_{i}\right)=\sum_{T_{k} \in P^{\circ}}{ }_{i} \operatorname{Prét}\left(P_{i}, T_{k}\right) \cdot v_{k}$, emptying speed of the place $P_{i}$ zero[6].

The difference: $B_{i}=I_{i}-O_{i}$ represents the dynamic balance of place $P_{i}$. $B_{i}$ can be positive, negative or

Property 1: The crossing speed of a strongly validated transition $T_{j}$ is equal to its maximum crossing speed:

$$
v_{j}(t)=V_{j} .
$$

Property 2 The crossing speed of a weakly validated transition $T_{j}$ is given by the relation:

$$
v_{j}(t)=\min \left[V_{j}, \min \left(B_{i}+v_{j}(t)\right)\right]
$$

With $i$ such that $P_{i}$ is a place of entry of $T_{j}$ and $m_{i}(t)=0$, [6]

\section{Application}

\subsection{Functional description of the system}

The material flow management linking three equipments: dosing feeder, rotary kiln and cooler, requires a reliable regulation, to ensure optimal production, a constant flow and a stable production line. The doser ensures a raw material for the rotary kiln with a variable flow set point, the production of the clinker deduced through a cooking process in the rotary kiln, enters directly into the cooler and forms a uniform pile, where it is cooled continuously by introducing a flow of ambient air.

Control and protection requires the preservation of equipment by measuring means, so as to ensure a dead pile in the cooler to protect it. The presence of temperature and pressure sensors in the cooler chamber and in the evacuation grids ensures a longer service life for production equipment.

\subsection{Clinker production process}

The production of clinker is ensured by a manufacturing process linking a set of equipment correlated with each other such as: material doser, rotary kiln, coolerand conveyor for evacuation and storage the clincker after cooling, it is simplified by the following synoptic, as shown in Figure 1. This process is 
managed by a control system, integrated in the programmable logic controller (PLC) to manage and control with a correction using the feedback of the measured information (feedback).

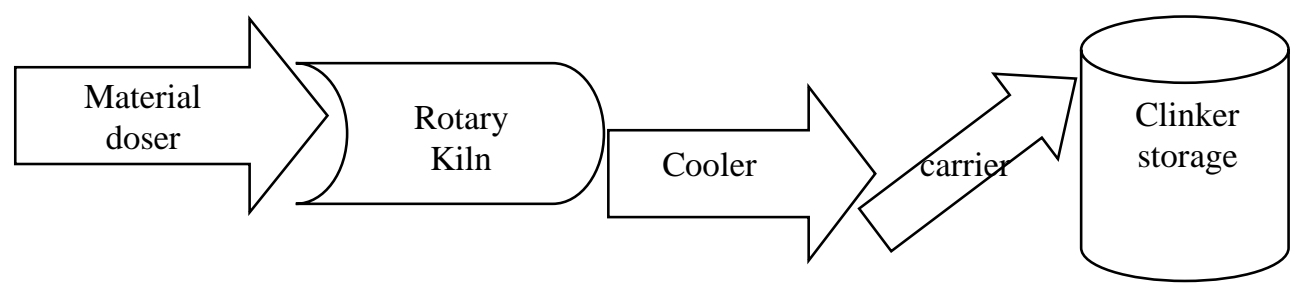

Figure 1. Production process: rotary kiln-cooler-clinker

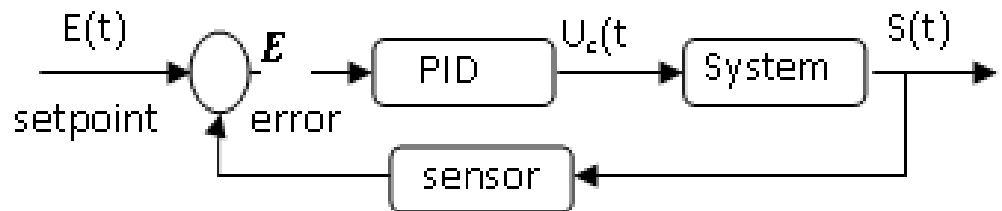

Figure 2. Closed-loop control system

Closed-loop control plays an important role in process control, in order to guarantee a dynamic and static behavior of the processes according to the defined specifications. It consists in maintaining a physical quantity to be adjusted, equal to a desired value, called a setpoint (Figure 2). This is achieved by adjusting and adapting the parameters of the transfer function of the process to be controlled. Closed-loop control is desirable when there are unpredictable changes in the parameters.

The control structure achieved thus makes it possible to improve the dynamic performance of the controlled system (speed, disturbance rejection, good setpoint tracking, sensitivity to parametric variations of the model, stabilization of unstable systems in an open loop).

\subsection{Components of the system}

The system under study consists of the following equipment (Figure 3):

- The Doser: is a device that from a requested flow rate will regulate the amount of material that feeds the rotary kiln from a storage hopper, an extraction system to manage the flow of material to a variable speed with a flow rate that varies from 0 to 150 ton per hour.

- Rotary kiln: The preheated material enters upstream of the rotary kiln and progresses to the clinkerization zone at a temperature of about $1400{ }^{\circ} \mathrm{C}$ to have a semi finished product(Clincker) at the outlet. The rotary kiln is made of sheet steel; it is protected inside by a refractory brick coating, slightly inclined and rotates at a variable speed proportional to the variable flow recorded in the doser to facilitate the flow of the product.

- Cooler: the clinker leaving the rotary kiln must be rapidly cooled in a cooler, from the temperature of the order of $1000{ }^{\circ} \mathrm{C}$ to about $100{ }^{\circ} \mathrm{C}$. This action is performed by injection of ambient air flow into the cooler through the pile of product in the evacuation phase; the excess hot air is discharged to the outside by a draft fan after a dedusting operation.

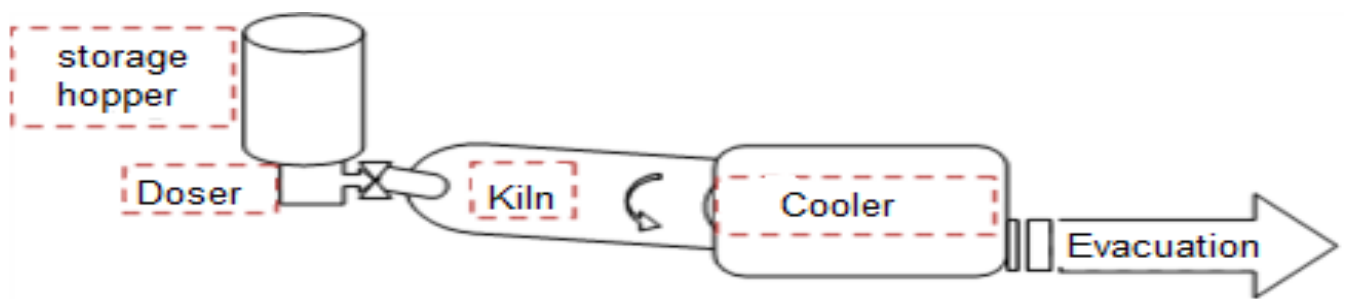

Figure 3. Overall scheme of a production system Rotary kiln-cooler

The cooler is controlled by a set of temperature sensors in order to ensure optimum cooling and manage injected cooling air volumes. The pressure measurement in the cooler can give a proportional image of the amount of clinker coming from the kiln and accumulated inside according to the evacuation speed. This measurement is given by a pressure sensor where can set minimum and maximum thresholds which represent the filling levels; they can be fixed here to a range that varies from 20 to 40 mbar. 
This pressure measurement can therefore be used to control the rate of discharge of the clinker in order to avoid the filling of the cooler, the overflow, the wear of the walls of the equipment and to ensure the stability of the rotary kiln, while eliminating repeated shutdowns of equipment which are the cause of the degradation of the production system. If the cooler is charging the chamber pressure increases and the speed must be increased for a quick and proportional discharge of the loaded clinker. When the clinker level decreases, the pressure also decreases and the speed must take the minimal value to maintain a low level of clinker.

\subsection{Flow adjustment}

The regulation of the flow consists in constantly checking the parameters involved in obtaining the calculation of the latter, namely the weight and the speed.

The material flow $D$ is given by :

$$
D=Q \times V(t / h), \text { Charge } \times \text { Speed }(\mathrm{Kg} / \mathrm{m} * \mathrm{~m} / \mathrm{s})
$$

The load is measured by a weight sensor, while the speed is obtained by a speed sensor.

\section{Model Design}

The design of the production line management model is realized by the application of the hybrid Petri nets in the Visual Object Net ++ software (Figure 4), which is a platform intended to model the dynamic hybrid systems by applying the formalism of the Petri net.

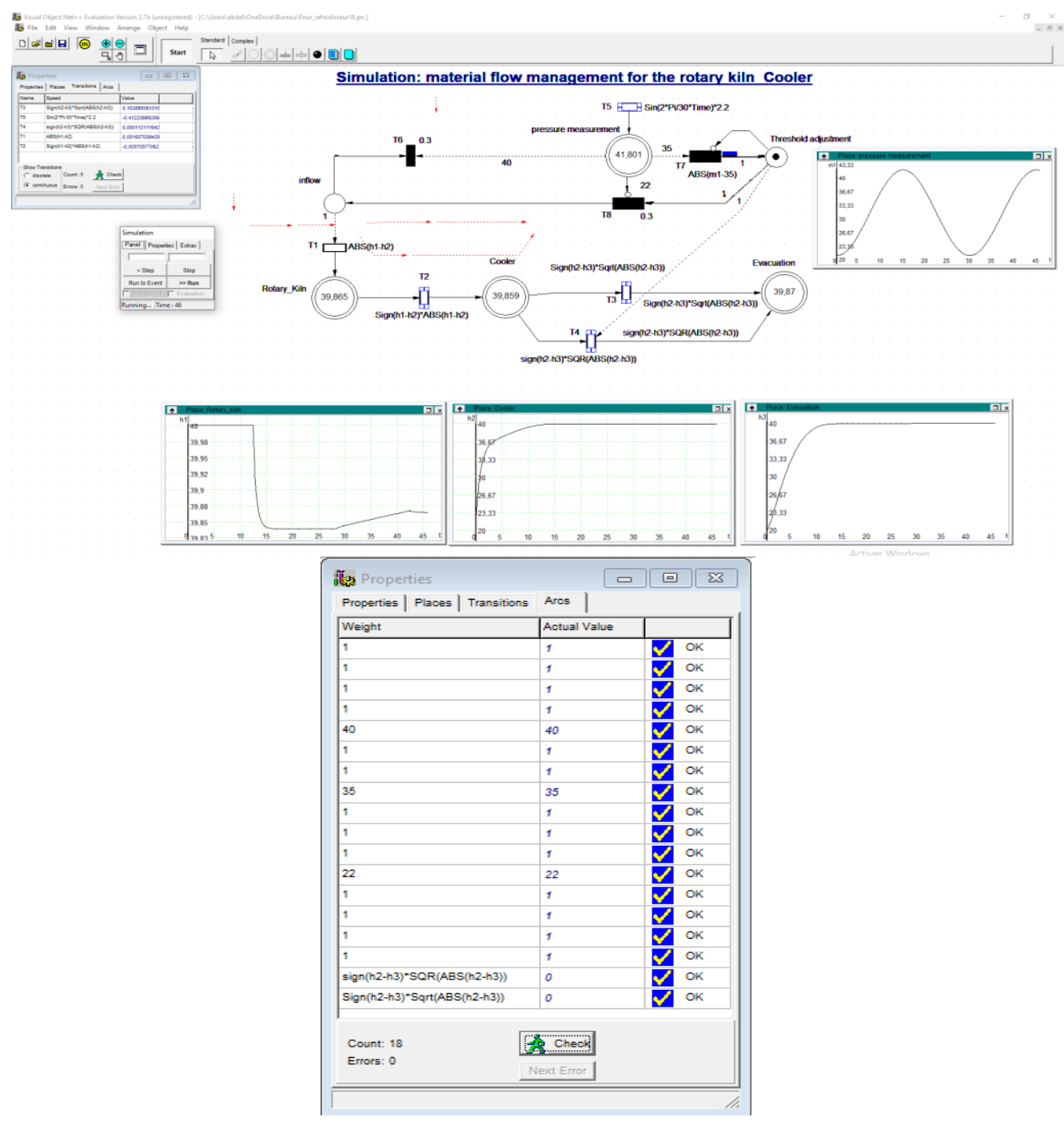

Figure 4. Simulator Software 'Visual Object Net ++', general view 


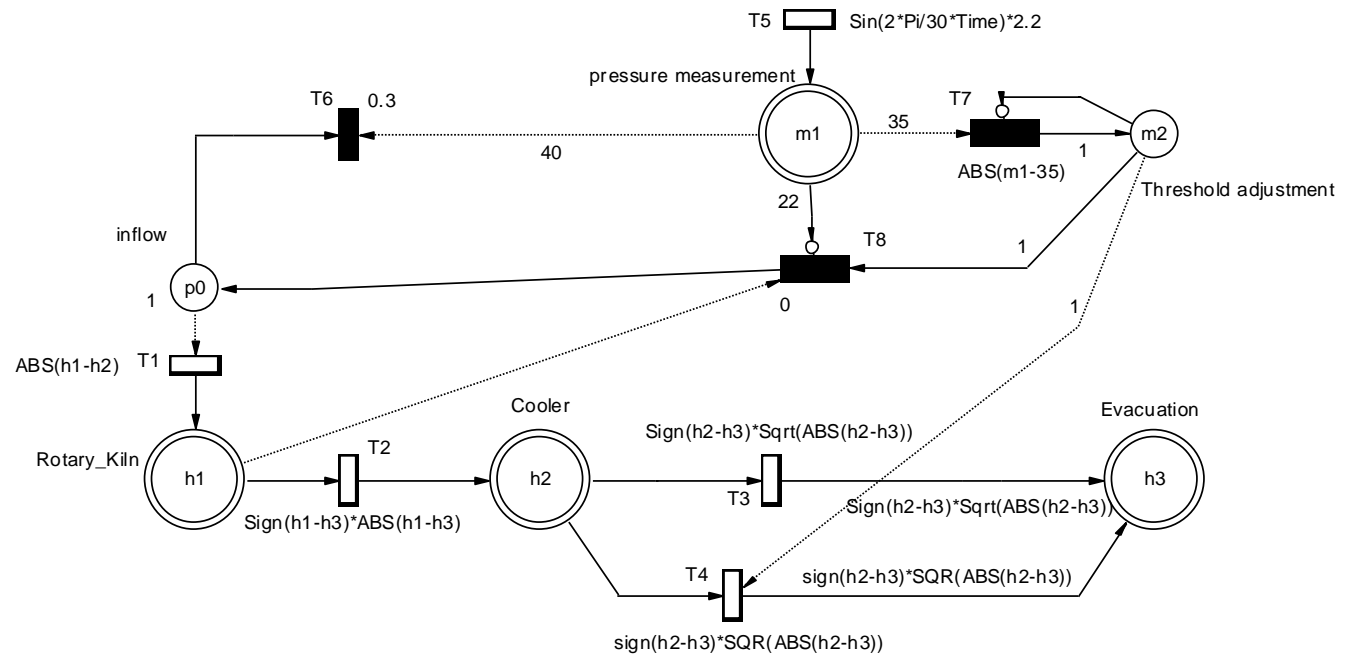

Figure 5. Global model of flow management system 'Rotary kiln-cooler'.

Figure 5 gives a description of the model for the proposed exhaust system: rotary kiln-cooler. The rotary kiln (place $: h_{1}$ ) is fed continuously by a semi finished product flow, provided by the doser (place: $P_{0}$ ) in order to produce clinker with a recorded flow rate that varies from 0 to $150 \mathrm{t} / \mathrm{h}$, the transition $T_{1}$ controls the flow of the doser with the predefined speed $V_{1}(t)$ :

$$
V_{1}(t)=\mid\left(h_{1}(t)-h_{2}(t) \mid\right.
$$

The transition $T_{2}$ controls the flow of the material in the kiln by the predefined speed $V_{2}(t)$ :

$$
V_{2}(t)=\left(h_{1}(t)-h_{3}(t)\right) \times \mid\left(h_{1}(t)-h_{3}(t) \mid\right.
$$

The cooler (place: $h_{2}$ ) receives the clinker from the furnace and ensures its evacuation cooled to a temperature of the order of $100^{\circ} \mathrm{C}$, it is characterized by the transitions $\mathrm{T}_{3}$ and $\mathrm{T}_{4}$ which ensure the continuous flow at two predefined speeds $\mathrm{V}_{3}(\mathrm{t})$ et $\mathrm{V}_{4}(\mathrm{t})$, to avoid filling and overflow of the material.

$$
\begin{aligned}
& \mathrm{V}_{3}(\mathrm{t})=\left(h_{2}(t)-h_{3}(t)\right) \times \sqrt{\left|\left(h_{2}(t)-h_{3}(t)\right)\right|} \\
& \mathrm{V}_{4}(\mathrm{t})=\left(h_{2}(t)-h_{3}(t)\right) \times\left(\left|\left(h_{2}(t)-h_{3}(t)\right)\right|\right)^{2}
\end{aligned}
$$

The transport system (place: $h_{3}$ ) ensures the evacuation and storage of the clinker leaving the cooler. The pressure sensor is represented by the place $m_{1}$, it gives the measurement of pressure in the cooler chamber, which varies from 20 to $40 \mathrm{mbar}$, the pressure is represented in the transition $T_{5}$ by a sinusoidal shape to give an image of the variation from the pressure of a minimum value to a maximum value, and it is given as follows:

$$
P=2,2 \times \sin \left(2 \times \frac{\pi}{30} \times \mathrm{t}\right)
$$

The place $m_{2}$ provides control of the system through two pressure adjustment thresholds $P_{1}$ and $P_{2}$ at transitions $T_{7}$ and $T_{8}$, they represent two levels of high and low fills in the cooler chamber. $P_{1}$ Is set to a pressure value for the low level of 22 mbar, while $P_{2}$ is set for the high level to 35 mbar, these two thresholds ensure the validation of the two preset speeds in $V_{3}(\mathrm{t})$ et $V_{4}(\mathrm{t})$.

A third threshold $P_{3}$ for safety is set to a value for the very high level to ensure the triggering and momentary shutdown of the doser through the discrete transition $T_{6}$, it is set to $40 \mathrm{mbar}$. This triggering of $P_{0}$ is reactivated by the transition $T_{8}$ if the pressure decreases at the level $P_{2}$.

\section{Simulation and results}

The execution of the simulation for the model chosen in the software simulator Visual Object Net ++ , with the parameterization and the adjustment of the properties by acting on the structure and the parameters of the model in the design phase and during the tests, in order to achieve to a reliable and stable solution of the dynamic system of the representative model (Figure 6).

This simulation describes the behavior of the dynamically evolving system with continuous flow velocities defined in the transitions $T_{1}, T_{2}, T_{3}$, and controlled by a discrete system represented by the place $\mathrm{m}_{2}$ which validates the crossing of the transitions $T_{3}, T_{4}$ (speeds: $V_{3}(t)$ and $V_{4}(t)$ ), respectively proportional to the pressure thresholds $\left(P_{1}, P_{2}\right)$ and disables the control of the place $P_{0}$ at the very high threshold $P_{3}$, in order 
to ensure a constant material flow and stable throughout the system. Figure 6 shows the settings and properties for the places and transitions for the discrete and continuous hybrid system, with the preset speeds to manage the flow of the proposed model.
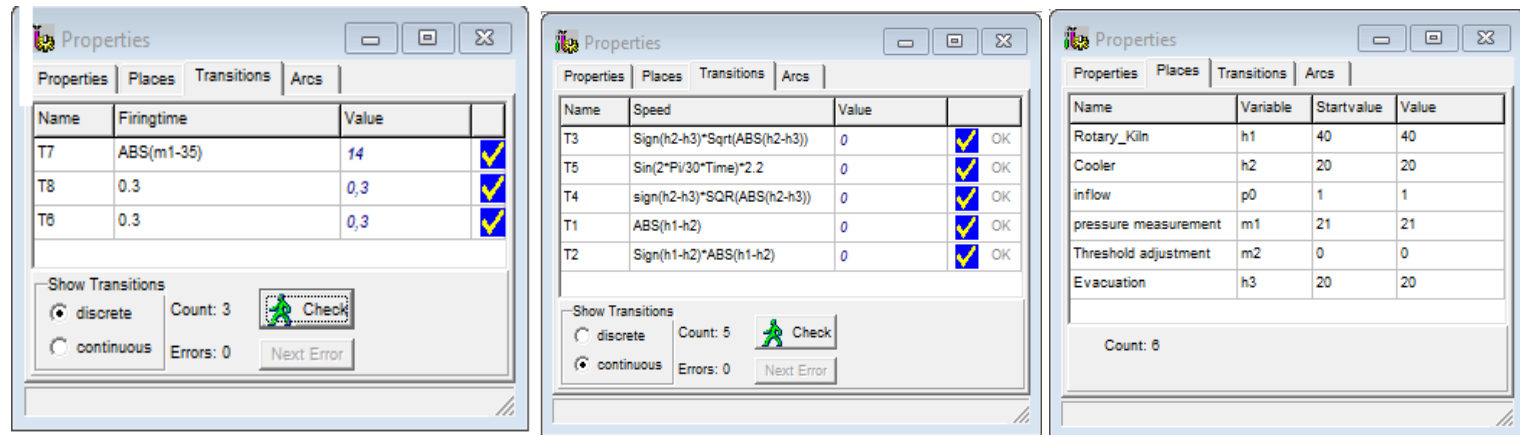

Figure 6. Adjustment properties and simulation parameters: Places,Transition (continuous and discrete) and Arcs

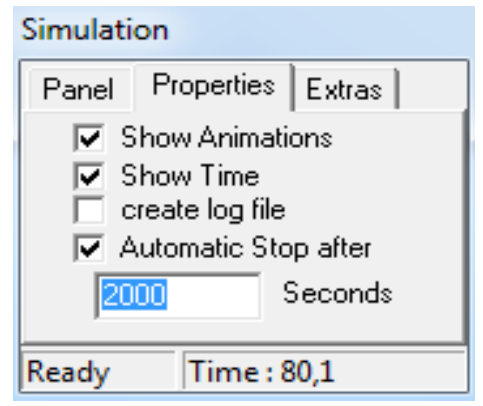

Figure 7. Setting and executing the simulation of the model

After setting the thresholds and setting the model, the simulation is started, as whown in Figure 7. The simulation starts the execution with the initial setting parameters (Figure 6). The control of the whole system "'Doser, Kiln, Cooler" with the evacuation system runs sequentially, with a correlation for the purpose to manage the flow of material discharged by the doser and ensure its final evacuation in a continuous manner.

The system validates transitions $T_{1}, T_{2}, T_{3}$ at initial velocities $V_{1}, V_{2}, V_{3}$, respectively, taking into account the pressure measurement of the cooler which varies from 22 to 35 mbar (Figure 8 ). This pressure gives a filling image and helps with a stable and continuous evacuation. If the pressure measurement has the value of the high pressure threshold $P_{2}=35 \mathrm{mbar}$ (Figure 9), the cooler begins to fill, and the system validates the operation of the second clinker discharge speed in the cooler $V_{4}$ with the speed $V_{3}$, hence the validation of transitions $T_{3}$ et $T_{4}$ at the same time.

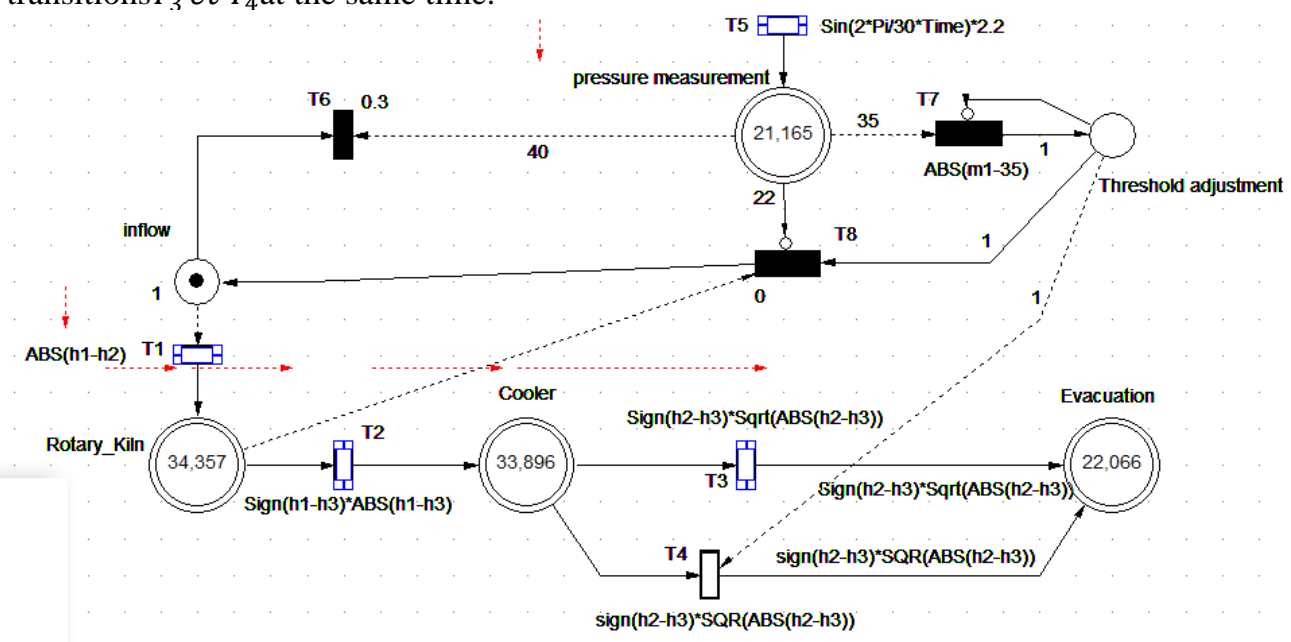

Figure 8 . The pressure threshold between $P_{1}=22 \mathrm{mbar}$ and $P_{2}=35 \mathrm{mbar}$ 


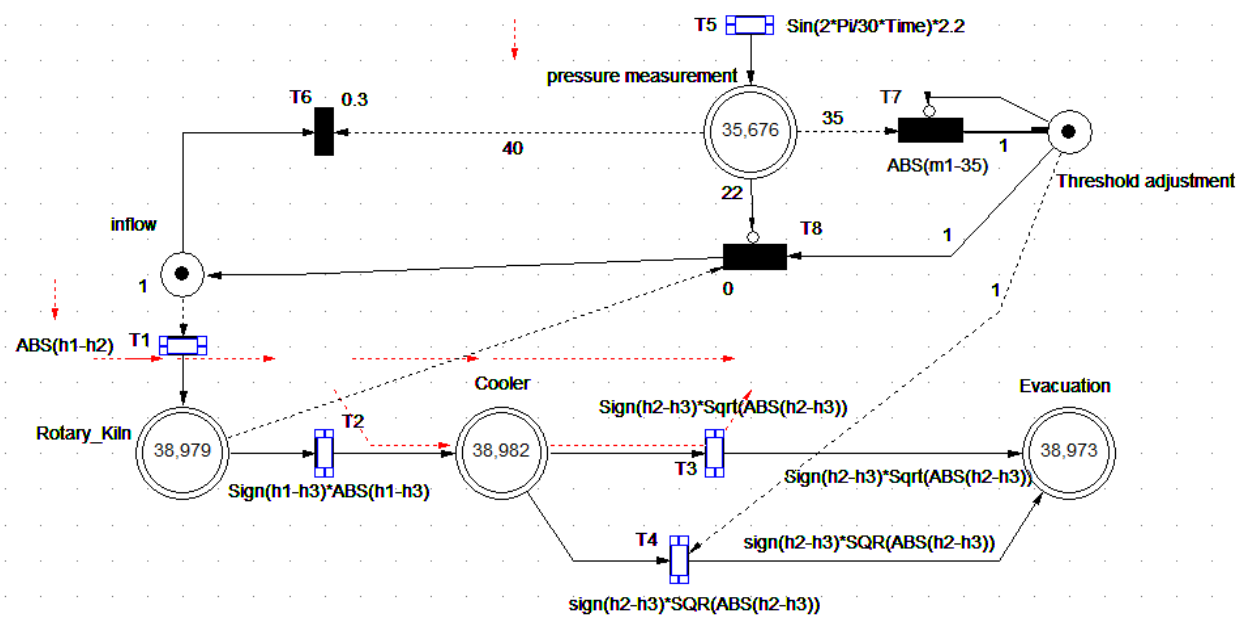

Figure 9. The threshold reaches the high level $P_{2}=35 \mathrm{mbar}$

If the measurement of the pressure exceeds the high threshold $P_{2}=35 \mathrm{mbar}$ and reaches the second very high threshold of the measured pressure $P_{3}=40$ mbar (Figure 10) in the cooler, this implies filling at a very high level, the system then blocks momentarily the $\operatorname{doser} P_{0}$ to relieve the flow rate in the kiln, and gives the cooler the necessary evacuation time with the preservation of validation of the two speeds $V_{3}$ et $V_{4}$, if the pressure decreases to $P_{1}=22 \mathrm{mbar}$, The system returns to normal operation by keeping the logged flow rate and the preset settings.

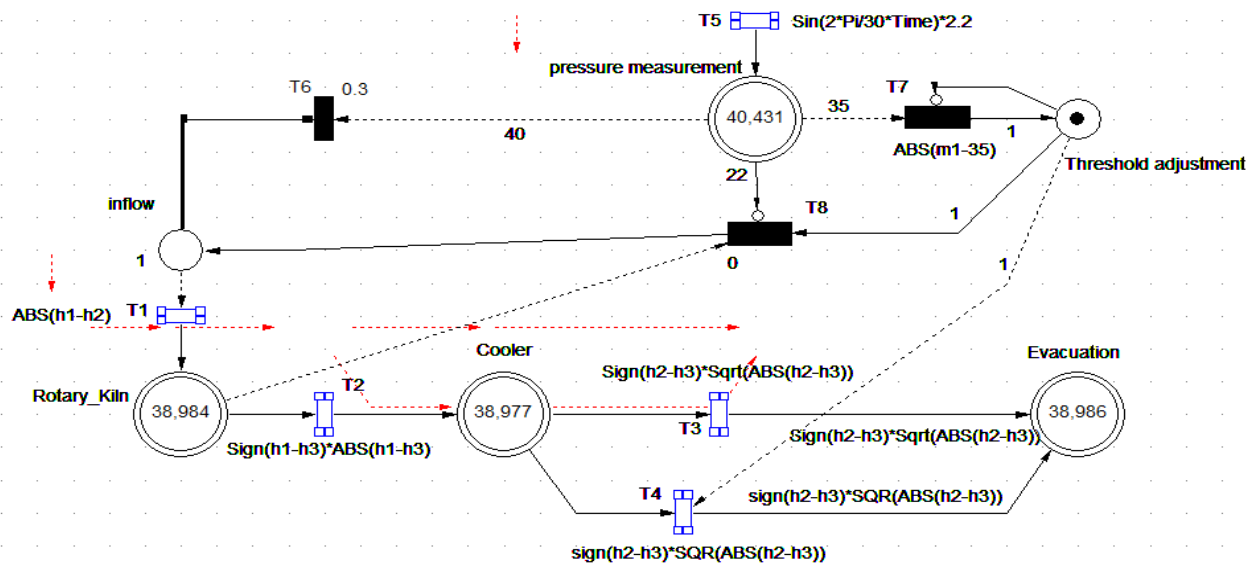

Figure 10. The threshold reaches the very high level $P_{3}=40 \mathrm{mbar}$

Figure 11 gives a representation to simulate the pressure value measured by a pressure sensor, and which varies from a minimum value to a maximum value (from 22 to 40 mbar) depending on the cooler filling. The setpoint monitoring in the kiln is ensured with stability and accuracy of flow $(\mathrm{t} / \mathrm{h})$, the system follows the setpoint, applying the choice and the setting of the evacuation speeds (Figure 12) and in respecting the nominal flow rate dedicated to equipment.

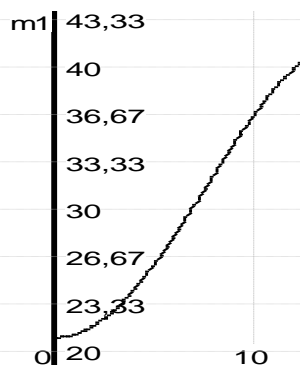

10

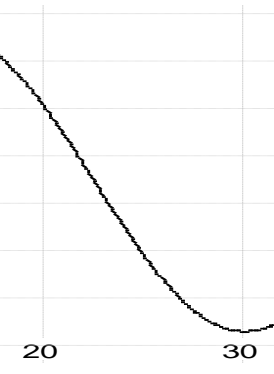

30

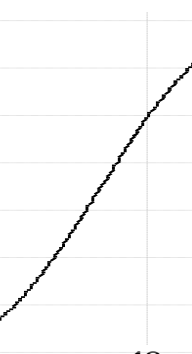

40

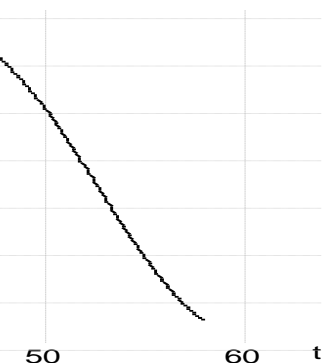

50
$60 \quad t$

Figure 11.Pressure measurement in the coolerwhich varies from 22 to 35 mbar 

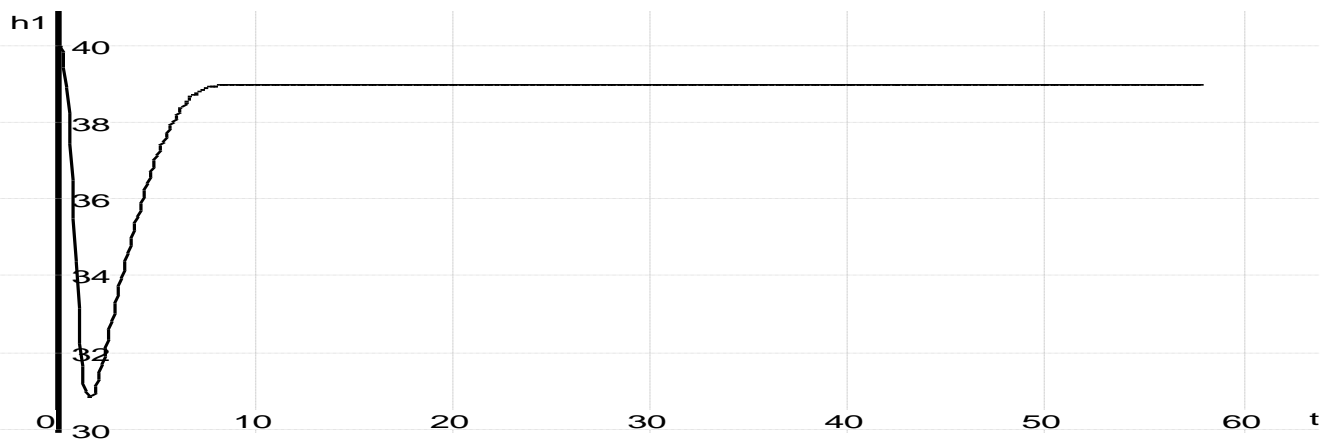

Figure 12. Variable flow setpoint in the place rotary_Kiln in (ton per hour)

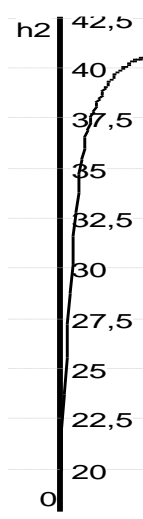

Figure 13. Clinker flow in the cooler (ton per hour)

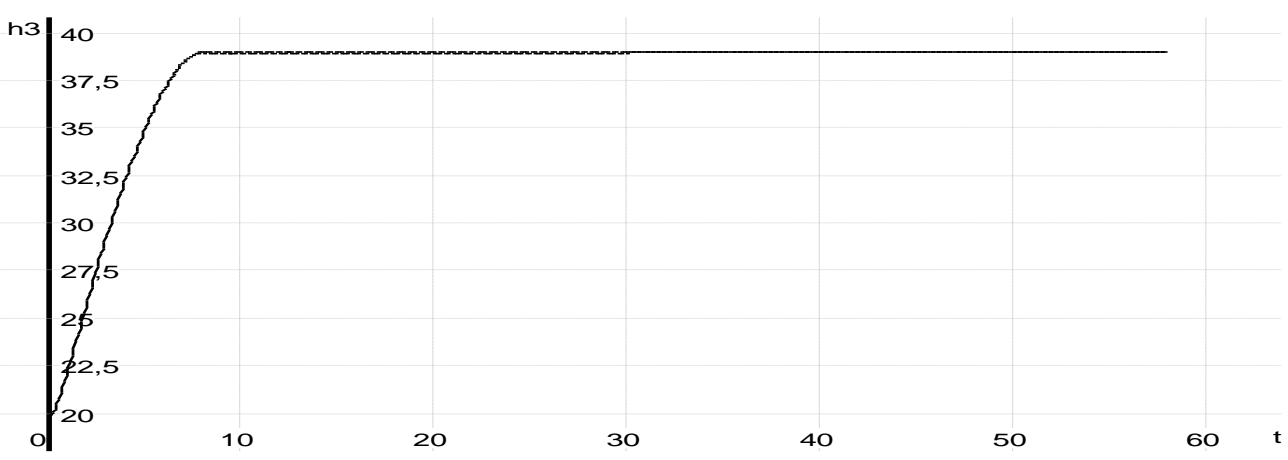

Figure 14. Clinker flow evacuate (cooler outlet)

The flow of the clinker into the cooler is maintained at the production rate in the kiln, and follows the changes in setpoint with each increase in the kiln flow rate (Figure 13). The evacuation system $\mathrm{h}_{3}$ ensures continuous flow to avoid jams, by following the flow of material from the Kiln-cooler system (Figure 14), and the adjustment of the evacuation velocities in coherence with the whole dynamic system.

This material flow management simulation shows us the behavior and dynamic evolution of the system which monitors and maintains a desired setpoint of the dosing flow rate upstream of the Kiln with a continuous and stable flow rate at the cooler outlet. The setting takes into account disturbances that can cause material damage in the equipment, because the same quantity of input must be discharged with a maintenance of a stable dead pile in the production circuit, the flow control according to the pressure measured in the cooler gives us information on whether or not the cooler is filled,this pressure value is verifiable as long as a lot of protection is guaranteed inside the cooler and this value is taken as a stable operating reference for the regulation.

\section{Conclusion}

Automation and regulation of industrial systems offer an opportunity for improvement in terms of speed of execution and adjustment. The design of the model with simulation on software simulators allows easy parameterization by acting on the structure and properties of the model plus correct operation of the system before proceeding with the final programming of actions. Research in modeling and flow 
management plays a key role in the design and evolution of industrial technology; it helps in construction and gives benefits with more efficient solutions, and more relevant for complex production lines.

Flow management is applied in the observation, control and monitoring of many dynamic industrial systems, in order to develop control actions and remedy them by continuous corrections, in order to improve and optimize the performance of these systems. Modeling by hybrid Petri nets is used in the control of hybrid dynamic systems. We can associate the markings of each place with control actions appropriate to the processes, on the other hand the events are carried out by these actions. As a result, they aid in real-time decision making in the control of complex industrial systems.

this study is based on the application of the formalism of hybrid Petri nets for the modeling of a hybrid dynamic system where the material flow is a continuous system and the control actions in discrete event to control the actuators in real time in order to " increase or decrease the evacuation speed. The essential interest lies in their analytical capacities through representations by graphic models. The validated analysis of the results makes it possible to extract from the model the relevant properties for the represented physical system in order to be managed appropriately. The simulated model can then be implemented on a computer in the form of a program or a database entrusted to an executable program.

The Kiln-Cooler system is fed by a metering device; it ensures a stable material flow (continuous system) and a regulatory dosage of finished and semi-finished products. This flow function is achieved by an interaction between the measured load and the continuous speed variation, the control is carried out by a discrete system which controls the opening and closing of the metering valve, which represents a hybrid dynamic system.

Controlling the discharge speed in the chiller provides rapid reaction to stabilize the system; it saves energy and increases the service life of equipment, minimizing repeated shutdowns and clincker overflow in order to optimize the production line. This work remains a platform for modeling and designing dynamic hybrid systems, and even in the tutorial plans, the use of powerful graphical tools makes it possible to automate and optimize complex systems that require more precision in the configuration before to be validated. Analysis and simulation are carried out on the "Visual Object Net $++"$ software simulator.

\section{REFERENCES}

[1] AD. Mircea, "Application of formalism Petri networks for the modeling of hybrid systems", in: International Conference on Electromechanical and Power Systems (ICEPS), University of Craiova, Faculty of Electromechanics, pp. 4-6, (October 2007).

[2] R. David, H. Alla, "On Hybrid Petri nets, Discrete Event Dynamic Systems: Theory and Applications", No.11,(2001), pp.9-40.

[3] J.Zaytoon,"Hybrid dynamic systems", IC2 Automated Systems Treaty, Ed. Hermès Science Europe, LTD Paris, (2001).

[4] René David, Hassane Alla "Du Grafcet aux Réseaux de Petri ", Edition Hermès Paris (1992), second édition.

[5] Melha Bitam, Hassane Alla, "Hybrid Petri nets in communication networks: transmission dynamics and behavioral study", JESA, Lavoisier, pp.73-94, (2006).

[6] Melha Bitam, "Modélisation et étude de comportement d'une ligne de communication TCP/IP", Thesis, Automatique/Robotique, Université Joseph-Fourier - Grenoble I, (2005).

[7] René David, Hassane Alla, "Discrete, continuous, and Hybrid Petri Nets", second edition, Springer. (2010).

[8] M. Allam, H. Alla, "Modelling Production Systems By Hybrid Automata and Hybrid Petri Nets", IFAC, Control of Industrial Systems, Belfort, France, (1997).

[9] H. ALLA. "Petri nets: A tool particularly suited to the modeling of hybrid systems", ADPM94, Bruxelles,Novembre(1994).

[10] Tolba C., Lefebvre D., Thomas P., Almoudni A.2005 «Continuous and timed Petri Nets for themacroscopic and microscopic traffic flow modelling”. Simulation Practice and theory vol $13 \mathrm{n}^{\circ} 5 \mathrm{pp} 407-436 \mathrm{Juillet} 2005$.

[11] NaitSidi-Moh A, Manier M.A, Elmoudni A, Wack M., 2006 « Petri Net with conflicts and Max+algebra for transportation systems » 11th IFAC Symposium on control transportation systems Delft Netherlands August 2006.

[12] Di Febrarro A, Giglio D, Sacco N 2004 « Urban traffic control structure based on hybrid petri nets » IEEE Transactions on intelligent Transportation Systems, Vol 5 n 42004 p 224-237.

[13] Dotoli. M, Fanti M.P, 2004 “An urban traffic network model via coloured timed Petri Net" WODES04 7th workshop on Discrete Event Systems September 04 Reims France.

[14] Moody J., Yamalidou K., Lemmon M., Antsaklis P. 1994 "Feedback control of Petri nets basedon place invariants", in Proceedings of 33rd Conference on Decision and Control, Lake Buena Vista, FL, pp. 3104-09.

[15] Lighthill et al. 1955, 'Contribution à la modélisation et à la simulation hybride du flux de trafic', thèse, EL HMAM Mohamed Said

[16] Industrial process documents for rotary kiln and cooler (cement industry)

IJEEI, Vol. 9, No. 1, March 2021 : $265-275$ 


\section{BIOGRAPHY OF AUTHORS}

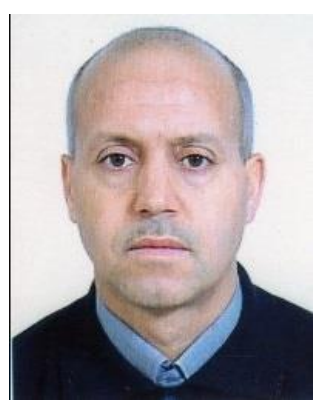

Mr Abdelkader Sayah

PhD student in AUTOMATIC

Laboratory of Automatics and Systems Analysis (L.A.A.S)

Department of Electrical Engineering, National School of Polytechnic Oran, Algeria.

Head of department Automatism and control measurement in cement industry, Algeria.

émail : abdelkader_saida@yahoo.fr

Professeur Mohammed Chenafa

Advanced control of complex dynamique systems

Laboratory of Automatics and Systems Analysis (L.A.A.S)

Departement of electrical engineering, ENP d'Oran B.P 1523, El Mnaouer, Oran, Algeria émail: mchenafa@yahoo.fr 